\title{
2 :RÉFORME DE LA COMPTABILITÉ ET MESURE DE LA PERFORMANCE DES AGENCES DU GOUVERNEMENT DES PAYS-BAS
}

Johan A. M. de Kruijf et Nico P. Mol

E.N.A. | Revue française d'administration publique

$2007 / 3-n^{\circ} 123$

pages 301 à 321

ISSN 0152-7401

Article disponible en ligne à l'adresse:

http://www.cairn.info/revue-francaise-d-administration-publique-2007-3-page-301.htm

Pour citer cet article :

de Kruijf Johan A. M. et Mol Nico P., « 2 :Réforme de la comptabilité et mesure de la performance des agences du gouvernement des Pays-Bas »,

Revue française d'administration publique, 2007/3 n 123, p. 301-321. DOI : 10.3917/rfap.123.0301

Distribution électronique Cairn.info pour E.N.A..

(c) E.N.A.. Tous droits réservés pour tous pays.

La reproduction ou représentation de cet article, notamment par photocopie, n'est autorisée que dans les limites des conditions générales d'utilisation du site ou, le cas échéant, des conditions générales de la licence souscrite par votre établissement. Toute autre reproduction ou représentation, en tout ou partie, sous quelque forme et de quelque manière que ce soit, est interdite sauf accord préalable et écrit de l'éditeur, en dehors des cas prévus par la législation en vigueur en France. II est précisé que son stockage dans une base de données est également interdit. 


\section{COMPTABILITÉ ET ACTION PUBLIQUE 2 :}

\section{RÉFORME DE LA COMPTABILITÉ ET MESURE DE LA PERFORMANCE DES AGENCES DU GOUVERNEMENT DES PAYS-BAS ${ }^{1}$}

\author{
Johan A.M. DE KRUIJF \\ Professeur assistant, Université de \\ Twente, Enschede, Pays-Bas
}

\author{
Nico P. MOL \\ Professeur, Université de Twente, \\ Enschede, Pays-Bas
}

Au sein de l'administration centrale des Pays-Bas, une comptabilité d'engagement est appliquée à certaines agences d'exécution dans le but d'en améliorer l'efficacité. À l'inverse du système comptable traditionnel de l'administration fondé sur une comptabilité de caisse, cette comptabilité d'engagement devrait permettre d'évaluer les résultats, les bénéfices moins les coûts, tirés de l'activité des agences. Cette réforme du système comptable n'a pas cependant modifié la relation hiérarchique qui existe entre, d'une part, les agences et, d'autre part, l'administration et les responsables politiques. Le statut des agences peut, ou non, permettre un certain degré d'autonomie dans les opérations, indépendamment du nouveau système comptable lui-même, mais il ne constitue pas une indépendance comparable à celle que détiennent les organismes publics indépendants qui ne sont pas liés au gouvernement par une relation de type hiérarchique ${ }^{2}$.

\section{LES AGENCES GOUVERNEMENTALES AU SEIN DU GOUVERNEMENT NÉERLANDAIS}

\section{Origine}

Au début des années 1980, l'administration centrale néerlandaise a traversé, comme bien d'autres administrations centrales, une crise financière. Afin d'y faire face et de maîtriser les dépenses publiques, la privatisation de certaines tâches administratives qu'elle

1. Article présenté à la conférence «Un secteur public performant ? Le second débat transatlantique », qui s'est tenue du $1^{\mathrm{er}}$ au 3 juin 2006 à Leuven et a été organisée par le Groupe européen d'administration publique (avec la collaboration de l'Instituut voor de Overheid, Katholieke Universiteit Leuven).

Traduit de l'anglais par Antoine Vauchez. Traduction revue par Sylvie Trosa.

2. Pollitt (C.), Talbot (C.), Caulfield (J.) and Smullen (A.), Agencies. How governments do things through semi-autonomous organizations, Palgrave, Basingstoke, 2004, p. 76. 
effectuait a été évoquée. La privatisation devait conduire à sous-traiter à des entités privées certaines activités qui étaient jusque-là effectuées par des institutions publiques ${ }^{3}$. Un grand nombre d'autres activités, telles que la délivrance de permis, fut considéré comme relevant naturellement de l'administration qui devait en conserver l'entière responsabilité. La possibilité d'une sous-traitance fût d'emblée écartée dans ces cas-là. Pour autant, la pression pour une plus grande efficacité de l'action publique s'est maintenue et, de ce fait, des alternatives à la privatisation ont dû être explorées. À partir de 1983 des expérimentations de contrats de management qui donnaient davantage de responsabilité aux dirigeants administratifs ont été mis sur pied ${ }^{4}$. Ces expériences ont, dans une certaine mesure, été couronnées de succès, mais le système de dépense classique fondé sur la comptabilité publique est alors apparu, entre autres problèmes, comme un obstacle à un usage plus efficient des ressources. Au même moment, des discussions sur l'étendue du contrôle des politiques publiques et sur les dirigeants d'administration centrale se sont développées. Afin d'assurer une meilleure garantie d'efficience et d'effectivité de l'action publique, deux types d'action ont été considérées comme souhaitables : une plus grande séparation entre la conception et l'exécution des politiques publiques et une exécution confiée à un ensemble d'administrations resserrées et à des entités administratives d'exécution plus ou moins indépendantes, créées ad hoc. En elles-mêmes, ces idées n'étaient pas neuves. En Suède, la distinction entre administrations et unités d'exécution est en vigueur depuis près de 300 ans 5 et le gouvernement britannique expérimentait alors parallèlement des politiques similaires. En fin de compte, un rapport ministériel de $1991^{6}$ proposa d'introduire le concept d'agence gouvernementale aux Pays-Bas. En 1994, quatre agences furent créées ${ }^{7}$. À la fin de l'année 2004, 31 agences gouvernementales étaient opérationnelles avec un budget de 5,6 milliards et un personnel de plus de 40000 personnes ${ }^{8}$, soit environ $40 \%$ des employés publics. Entre autres exemples, on peut citer l'Agence des institutions pénitentiaires (1,8 milliards d'euros, 18300 employés), la Direction de l'immobilier public (1,3 milliards d'euros, 950 employés) et l'Autorité pour la nourriture et la sécurité des consommateurs (0,2 milliards d'euros, 1100 employés).

Dans la littérature internationale, le concept de Quangos (Quasi-Autonomous National Government Organisation) et celui d'agences gouvernementales sont souvent employés 9 . C. Talbot donne une brève définition de leurs caractéristiques. Une agence est une entité « à

3. Boorsma (P.B.) and Mol (N.P.), Privatisering, Stichting Maatschappij en Onderneming, 's Gravenhage, 1983 , p. $20-23$. 1998.

4. Mol (N.P.), Bedrijfseconomie voor de collectieve sector, $4^{\mathrm{e}}$ druk, Samsom/Vuga, Alphen aan den Rijn,

5. Statskontoret, What lessons can we learn form the UK's next steps agencies model?, 2001, http://www.statskontoret.se/upload/Publikationer/2001/200123.pdf.

6. Ministerie van Financiën (Heroverwegingswerkgroep Beheersregels), Verder bouwen aan beheer, SDU, Den Haag, 1991.

7. Le service d'immigration et de naturalisation (IND), l'organisation de défense et télématique, Senter-Novem et le service de protection des plantes (PD).

8. Toutes les agences gouvernementales ne publient pas leurs effectifs dans leurs rapports annuels : 38000 employés sont comptabilisés sur la base de ces seuls rapports d'activité. Pollitt (C.), Talbot (C.), Caulfield (J.) and Smullen (A.), Agencies. How governments do things through semi-autonomous organizations, Palgrave, Basingstoke, 2004, p. 76.

9. Flinders (M.V.) and Smith (M.J.) dir., Quangos, accountability and reform. The politics of Quasi-Government, Palgrave MacMillan, Houndmills/New York, 1999; OECD, Distributed Public Governance. Agencies, Authorities and other government bodies, (Les autres visages de la gouvernance publique), OECD, Paris, 2002 ; Zwart (T.) and Verhey (L.) dir., Agencies in European and Comparative law, Intersentia, Antwerp, 2003 ; Pollit (C.) and Talbot (C.), Unbundled Government, Routledge, Oxon, 2004 ; Pollitt (C.) and Bouckaert (G.), Public Management Reform. A comparative analysis, 2nd edition, Oxford University Press, Oxford, 2004. 
distance du cœur de la hiérarchie des administrations étatiques centrales et qui exerce des missions publiques à un niveau national ; elle est composée de fonctionnaires financés par le budget de l'État ou dont le financement demeure sous la responsabilité de l'État; elle reste enfin sujette au moins pour partie à des procédures de droit public ${ }^{10}$. Un Quango est généralement considéré comme plus autonome vis-à-vis de l'administration que ne l'est une agence gouvernementale ${ }^{11}$. Ces dernières n'ont pas de statut juridique distinct de l'administration; elles font partie intégrante de l'entité juridique étatique et restent inclues dans la structure même des administrations centrales. Mais elles disposent d'une position à part du fait de la relative autonomie de management dont elles bénéficient et du fait qu'elles utilisent d'une comptabilité d'exercice/d'engagement (accruals budgeting and accounting system) en lieu et place de la comptabilité de caisse (cahs/encumbrance based accounting system).

\section{Création}

Quand un ministre souhaite conférer à une entité de son administration le statut d'agence, il doit remplir un certain de nombre de requisit politiques et organisationnels. Considérons tout d'abord les conditions proprement politiques. Le ministre doit soumettre au Parlement son intention de mettre sur pied une telle structure ${ }^{12}$. Cette proposition est présentée selon une procédure souple; il s'agit d'une lettre dans laquelle le ministre fait part de son projet et des raisons qui le motivent. Cette lettre doit disposer du soutien de l'ensemble du gouvernement, y compris du ministre des finances. Le Parlement dispose ensuite de trente jours pour demander des informations complémentaires ou pour rejeter le projet. Quand il y a rejet, l'agence gouvernementale ne voit tout simplement pas le jour. Si aucune information n'est demandée ou si le Parlement est satisfait des informations complémentaires qui lui sont été fournies, alors l'agence gouvernementale est créée. L'expérience a montré que l'établissement d'une agence gouvernementale n'a été bloquée qu'une seule et unique fois pour des raisons essentiellement politiques. Il s'agit du projet de création du Belagstingdienst ou Service du revenu interne. Le Parlement avait craint que l'agence ne dispose de trop de liberté dans la mise en place de régulations sans accord politique préalable. Bien que l'argument du Parlement ne fut pas juridiquement correct, il touchait bien au cœur du débat politique sur la responsabilité ministérielle. Dans l'architecture constitutionnelle néerlandaise, un ministre est en effet responsable pour tous les actes qui ont été engagés sous son autorité. Cela inclut toutes les activités de l'administration centrale dont il a la charge. Dans la mesure où les agences gouvernementales font formellement partie des administrations, même sous une forme spécifique, le ministre est donc pleinement responsable pour les activités et les décisions prises au sein de l'agence gouvernementale. Ainsi, bien que ce cas de refus soit unique, il fait néanmoins comprendre où se situe l'inquiétude politique à l'égard des agences gouvernementales.

10. Talbot (C.), «The Agency Idea », in C. Pollit and C. Talbot, Unbundled Government, Routledge, Oxon, 2004, p. 5.

11. La même littérature internationale identifie en France trois types d'entités disposant d'une certaine autonomie l'égard de l'administration centrale, dont les autorités administratives indépendantes forment le cas le plus proche, au moins pour partie, des agences gouvernementales néerlandaises. Rochet (C.), Cabane (M.) and Formery (S.), «France», in OECD, Distributed Public Governance. Agencies, Authorities and other government bodies, OECD, Paris, 2002, p. 76-77 ; Heringa (A.W.), « Agencies in France », in Zwart en Verhey, Agencies in European and Comparative law, Intersentia, Antwerp, 2003, p. 41-47.

12. L'établissement d'une agence signifie ici la réorganisation interne de l'administration et non pas l'institution d'une entité dotée d'une statut juridique autonome. 


\section{Fonctionnement}

Cette prérogative du législateur, qui consiste à approuver les propositions de budget de l'administration, vaut également pour ce qui touche aux budgets des agences gouvernementales. Les lois de finances comprennent des budgets distincts: dépenses, revenus et investissements. Le Parlement approuve ces différents budgets séparément pour chacune des agences gouvernementales. La première différence avec les propositions budgétaires des administrations classiques tient au fait que les budgets des agences gouvernementales comprennent exclusivement les coûts opérationnels des entités exprimés en dépenses et non en charges. Les propositions budgétaires classiques sont fondées sur l'évaluation des charges qui comprennent tout à la fois les coûts du programme lui-même et ceux de sa mise en œuvre ; en outre, elles ne peuvent pas être directement perçues à un niveau administratif inférieur aux directions générales. La seconde différence tient au fait que le budget d'investissement d'une agence gouvernementale lui permet d'emprunter à l'administration des finances, laquelle lui fait alors payer les intérêts de son prêt. À l'inverse, les structures administratives ordinaires ne peuvent pas emprunter pour leurs investissements ; leurs investissements doivent être intégrés dans leurs budgets prévisionnels. Une dernière différence consiste dans le fait que le budget d'une agence gouvernementale n'est pas censé être re-discuté en cours d'année, les éventuels écarts par rapport aux budgets originels étant débattus exclusivement en fin d'année budgétaire. Au contraire les programmes budgétaires ordinaires des administrations sont, eux, soumis à une discussion de politique budgétaire à deux moments de l'année : une première fois à la fin du printemps et une autre à la fin de l'automne. À ces deux moments, les budgets peuvent être modifiés en fonction des résultats des choix politiques opérés.

En principe, les agences gouvernementales sont des entités chargées de remplir une tâche bien spécifique selon un cadre politique prédéterminé. Les activités des agences sont essentiellement routinières. Elles n'impliquent aucune activité de régulation pas plus qu'elles ne comportent de fonction quasi-juridictionnelle. Les agences sont essentiellement pensées pour améliorer l'efficacité du processus de production de l'activité administrative. De manière à atteindre cette meilleure efficacité, il a été considéré qu'une plus grande marge de manœuvre managériale était nécessaire dans l'usage des ressources. Cette plus grande latitude managériale permet également de recruter une équipe sur la base qui correspond le mieux aux besoins de l'agence elle-même. Tout en restant dans le cadre de l'échelle des rémunérations et des procédures de recrutement de la fonction publique, les dirigeants d'une agence peuvent décider, sans intervention d'autres autorités administratives, du recrutement ou de l'éviction de telle ou telle personne.

Bien que les conditions spécifiques pour établir une agence gouvernementale aient quelque peu changé au cours des années, les caractéristiques principales restent identiques : présence d'une «production» mesurable, usage d'une comptabilité d'exercice/ d'engagement, c'est-à-dire d'un système complet de comptabilisation des coûts (full-cost accounting), réelles opportunités d'amélioration de l'efficacité et pratique comptable se conformant aux standards établis en la matière par le droit. Dans un schéma idéal, la relation entre l'administration centrale et l'agence s'apparente à celle qui existe entre maître d'ouvrage et maître d'œuvre ${ }^{13}$. Mais ce schéma risquait grandement de perdre en efficacité et en clarté, car l'administration centrale n'était pas à proprement parler en position de maître d'ouvrage : l'agence ne lui était pas externe. Pour faire face à cette

13. Oosteroom (R. van), « The Netherlands », in OECD, 2002, Distributed Public Governance, OECD, Paris, 2002, p. 113-132. 
difficulté, il a été donné aux conseils d'administration des ministères le rôle de commanditaire négociant le prix et la qualité de la production fournie par l'agence gouvernementale. Les dirigeants du ministère jouent le rôle de propriétaires et contrôlent ainsi les résultats financiers et la continuité du travail de l'agence ${ }^{14}$.

Il est apparu difficile de mesurer la production actuelle et de développer des comptabilités de gestion (cost accounting systems). Pour autant, le fait de donner plus d'autonomie a renforcé l'estime et, partant, la motivation des personnels. Le recours aux agences gouvernementales est maintenant un fait politique établi. Il est difficile d'imaginer qu'à l'avenir le Parlement refuse l'établissement d'unités autonomes de ce type. Il y a pourtant des signes que ce mouvement d'autonomisation touche à sa fin. Début 2007, la plupart des unités qui pouvaient être inclues avait d'ores et déjà reçu le statut d'agence gouvernementale. Si le Belagstingdienst (Service du revenu interne) était finalement intégré dans le groupe des agences gouvernementales, ce seraient pas moins de $80000 \mathrm{em}$ ployés qui travailleraient pour ces agences, soit près de $75 \%$ des employés publics. Dans les administrations centrales ne restent désormais que les équipes chargées de la conception des politiques publiques elles-mêmes.

\section{PROBLÉMATIQUE}

La réforme du système comptable n'est pas en soi une condition suffisante pour imprimer aux agences des comportements typiques d'une entreprise privée. Les évaluations n'ont pas toujours fait apparaître une amélioration de l'efficacité ${ }^{15}$. La possibilité d'obtenir des «résultats financiers» mis en évidence par l'introduction du système comptable n'emporte pas, pour autant, automatiquement une gestion orientée vers résultats. Dans un cadre marqué par un double monopole de l'agence (production) et de l'administration (consommation), les ressources fournies à l'agence peuvent indifféremment être référencées comme « revenus » ou comme « ressources ». Dans un cadre marqué par un monopole de l'offre par une seule entité, les coûts peuvent être facturés sans conditions aux clients (externes). Dès lors, un travail empirique devrait permettre de faire apparaître dans quelle mesure la réforme des agences a effectivement contribué à l'amélioration de leur efficacité.

Nous allons tenter de voir, en nous appuyant sur deux enquêtes (1998 et 2002) relatives à la réforme des agences engagée en 1994, pourquoi les résultats sont ambigus ${ }^{16}$. Les enquêtes de 1998 et 2002 ont fait apparaître des doutes quant à l'efficacité effective des améliorations résultant de la réforme des agences. Ces dernières sont particulièrement conscientes des enjeux qui tournent autour de leur performance. Elles perçoivent clairement qu'une comptabilité d'engagement constitue une obligation de rendre des comptes quant à leurs «résultats ». Suivant en cela l'exemple des organismes publics indépendants, elles se sont en général engagées à produire des efforts pour se responsabiliser face aux partenaires et aux usagers. En effet, les agences sont incitées à fournir d'elles-mêmes les preuves de l'amélioration de leurs performances. Mais, contrairement à ces autres organismes publics, leur responsabilité à l'égard des partenaires extérieurs est

14. Blokhuis (M.) en Slootmaker (J.), « De rol van de eigenaar », in Kraak (A.D.) en Oosteroom (R. van) ed., Agentschappen : innovatie in bedriffsvoering, SDU, Den Haag, 2002, p. 104-111.

15. Berenschot, De evaluatie van het baten-lastendienstmodel, Eindrapport, Utrecht, 2002.

16. Le seul élément fourni par l'enquête de 2002 consistait dans la production d'indicateurs de performance indirects montrant que la mesure de celle-ci retenait désormais davantage l'attention. Assurément, cette attention peut simplement être la conséquence des objectifs formels inscrits dans le statut de l'agence (indiquant l'importance de l'évaluation de l'efficacité pour l'organisation). 
secondaire par rapport aux exigences fixées par leurs maîtres d'ouvrage principaux (la hiérarchie centrale): en d'autres termes, la responsabilité «partenariale » ne peut se substituer à la responsabilité hiérarchique que lorsque les agences deviennent effectivement indépendantes.

On pourrait soutenir, de manière théorique, que la neutralité compétitive entre le secteur public et le secteur privé et les relations contractuelles de type client-fournisseur sont des conditions de la performance. Mais rares sont les mises en œuvre pleines et entières de ces principes abstraits dans le cadre des relations entre l'administration et les agences. Habituellement, l'agence fournisseur reste un producteur centré sur un seul service et les restrictions posées à la concurrence vont au moins altérer les mécanismes de fixation des prix, l'absence de clients extérieurs risquant d'empêcher toute forme d'évaluation monétaire. Le contrôle budgétaire reste donc en vigueur et on doit dès lors se demander dans quelle mesure les agences peuvent néanmoins être gérées comme des centres de profit (business units).

M. Blokhuis et S. Slottmaker ${ }^{17}$ relèvent que la distinction entre propriétaire, maître d'ouvrage et maître d'œuvre est utile pour créer un quasi-marché des activités réalisées par l'agence. Cela peut aider à réduire l'importance du contrôle budgétaire. Nous avons observé que, dans un certain nombre de cas, le maitre d'ouvrage n'est pas le consommateur du service effectué. Ainsi, dans le cas des transferts de revenus (income transfers), celui qui a effectivement demandé un tel transfert est le consommateur, indépendamment de la question de savoir s'il doit, ou non, payer pour une telle requête. Il est clair que le maître d'ouvrage - qui paye pour cette tâche - aura davantage de difficultés à utiliser des instruments de contrôle budgétaire que si le maître d'ouvrage et le consommateur étaient une seule et même personne. En tout état de cause, il est nécessaire de prendre en compte les différents types de relations existant entre les maîtres d'ouvrage et leurs agences pour ce qui touche au contrôle du comportement de ces dernières. L'impact de l'introduction d'une comptabilité d'engagement dépendra d'une manière générale des caractéristiques de l'agence elle-même. Un premier effort pour échafauder une classification susceptible de saisir ces différents cas a d'ores et déjà été engagé. Elle est fondée sur les missions attribuées aux agences gouvernementales ${ }^{18}$. Nous n'avons pas trouvé de travaux dans lesquels cette classification ait pu être testée. On peut cependant relever que, d'après A. Smullen ${ }^{19}$, ce n'est que dans $23 \%$ des cas que la recherche de l'efficacité a constitué une raison pour la création d'une agence gouvernementale spécifique. Contrairement à ce qu'on pourrait croire, l'amélioration de l'efficacité ne constitue donc qu'un objectif secondaire de la réforme du système comptable des agences. Pour autant, l'introduction d'une comptabilité d'engagement peut contribuer à renforcer l'efficacité des agences. De notre point de vue, cela dépend moins au premier chef des missions assignées à l'agence que de leur réelle responsabilisation sur leurs résultats (revenus / coûts) inscrits dans leur comptabilité d'engagement. En théorie, le degré d'autonomie (libertés de gestion compétences discrétionnaires) attribué par les systèmes de comptabilité et de contrôle devrait suivre de près le degré de responsabilité des agences pour ce qui touche aux revenus

17. Blokhuis (M.) et Slootmaker (J.), « De rol van de eigenaar», op. cit., p. 104-111.

18. Smullen (A.) et Thiel (S. van), «Agentschappen : eenheid in verscheidenheid », in Kraak (A.D.) en Oosteroom (R. van) ed., Agentschappen : innovatie in bedrijfsvoering, 2002, SDU, La Haye, p. 41.

19. Smullen (A.), « Lost in translation ? Shifting interprÉtations of the concept of 'agency' : the Dutch case », in Pollitt (C.) and Talbot (C.) ed., Unbundled government, Routledge, Londres et New York, 2004, p. 190. 
et aux coûts. Et cette autonomie devrait en conséquence se retrouver au sein des agences elles-mêmes dans des modes de décision ayant le résultat financier pour objectif.

Nous commencerons donc par caractériser ces agences, qui peuvent toutes être considérées comme des centres de production, selon qu'elles constituent des centres de profit ou des centres de coût. En tant que centres de production, les agences peuvent relever de l'un ou l'autre de ces deux types de centre, avec une grande variété de formes de responsabilité entre les deux. Dans les centres « exclusivement » construits autour du profit, la comptabilité d'engagement peut être nécessaire pour évaluer les résultats (financiers) des revenus des ventes au regard des coûts de production. Dans les centres «exclusivement » construits autour du coût, les instruments budgétaires traditionnels sont sans doute mieux adaptés pour assurer un contrôle. Entre les deux, les contrats de service s'accompagnant de dispositions plus ou moins obligatoires, par exemple des accords sur les niveaux de service (service level agreements), entre les agences et leurs clients sont caractéristiques d'une budgétisation par les résultats appliquée aux agences.

\section{L'ANALYSE EN TERMES DE CENTRES À RESPONSABILITÉ COMPTABLE}

Notre travail sera mené en ayant recours une analyse en termes de centre à responsabilité comptable (responsibility accounting). La principale question que pose la notion de centre à responsabilité comptable est la suivante : dans quelle mesure un manager particulier peut-il être tenu pour responsable de la performance financière de l'organisation qu'il dirige 20 ? Plusieurs auteurs remarquent que la responsabilité comptable devrait être choisie dans l'esprit de permettre de futures améliorations de la performance plutôt que dans celui de pénaliser une gestion en cas de performance insuffisante ${ }^{21}$. Il existe quatre catégories de centres à responsabilité comptable.

La plus importante de ces catégories est celle du «centre d'investissement » au sein duquel le dirigeant est complètement responsable de tous les aspects, y compris les décisions d'investissements, du contrôle financier de l'organisation dont il a la charge. Dans un centre d'investissement, l'élément essentiel de la performance tient dans le retour sur investissement.

À l'inverse, la forme la plus restrictive est le «centre de dépense» ou, plus précisément, le centre de dépense discrétionnaire. C. Drury décrit ce type de centre comme une entité "où le résultat ne peut être mesuré en termes financiers et où il n'y a pas clairement de relation observable entre les ressources et les résultats » ${ }^{22}$. Il fait de l'unité de recherche et développement l'exemple typique de ces centres de dépense discrétionnaire. Dans ce cadre, le contrôle consiste, d'après C. Drury, à évaluer le rapport entre les dépenses effectuées et les dépenses budgétées puis à s'assurer que les missions assignées au centre ont été accomplies avec succès. La description d'un centre de dépense

20. Lapsley (I.), «Responsibility accounting revived ? Market reforms and budgetary control in health care », Management accounting research, Vol. 5, $\mathrm{n}^{\circ} 3-4,1994$, p. 337-352; Merchant (K.A.), Modern management control systems, Prentice Hall, NJ, 2000 ; Drury (C.), Management and cost accounting, 6th ed., Thomson, Londres, 2004, p. 663-665 ; Mol (N.P.), Operationele budgettering in de publieke sector, SDU, La Haye, 2006.

21. Garrisson (R.H.), Noreen (E.W.) et Brewer (P.C.), Managerial accounting, 11th ed., McGraw Hill, Boston, 2006 ; Horngren (C.T.), Datar (S.M.) et Foster (G.), Cost Accounting. A managerial emphasis, 12th ed., Pearson Prentice Hall, NJ, 2006, p. 199.

22. Drury (C.), Management and cost accounting, op.cit., p. 654. 
discrétionnaire ressemble ainsi fortement aux méthodes budgétaires traditionnelles utilisées par les administrations. La budgétisation par moyens, qui est utilisée par de nombreuses administrations, correspond très bien au modèle du centre de dépense discrétionnaire ${ }^{23}$. Il existe deux sous-types particulièrement importants du centre de dépense (discrétionnaire) : le centre de dépense - coût standard, d'une part, et le centre de dépense - coût complet (également appelé «centre de coût» tout court), d'autre part. Dans un centre de dépense - coût standard, le dirigeant est tenu pour responsable des coûts de production quand le résultat peut être évalué et qu'une description des moyens attendus pour chaque unité est disponible. Le contrôle est fondé sur des analyses des écarts entre les coûts budgétés par unité de produit et les coûts de production effectivement induits. C. Drury reconnaît, que dans un centre de coût standard, des contrôles supplémentaires (non financiers) doivent être mobilisés pour tout ce qui touche à la qualité du service offert ${ }^{24}$. Notons ici que des «tableaux de bord prospectifs» (balanced scorecards) peuvent être un moyen d'inclure des informations non financières dans les mécanismes de contrôle. Ces « tableaux de bord prospectifs » peuvent être très utiles pour chacun des types de centre de responsabilité. Cependant, quand le système budgétaire est fortement orienté vers le contrôle des dépenses, l'instrument principal de contrôle reste la marge (caseflow) plutôt que des indicateurs construits autour de l'évaluation de la performance tels qu'inclus dans les «tableaux de bord prospectifs ».

Entre ces deux extrêmes, il existe deux autres types. D'abord, le centre de profit qui est très proche d'un centre d'investissement. La principale différence entre les deux tient dans l'absence d'autorité du dirigeant d'un centre de profit pour décider des investissements. Selon K. A. Merchant, l'objectif d'un centre de profit est «normalement» d'atteindre un équilibre financier au niveau des profits budgétés ; les profits qui excédent ce qui avait été initialement prévu n'étant pas souhaitables ${ }^{25}$.

Vient ensuite le centre de revenu qui est, lui, plus proche du centre de dépense discrétionnaire. Le management d'un centre de revenu est essentiellement censé générer des revenus à la manière par exemple d'un service de ventes. Sous une forme plus sophistiquée, la direction est également tenue pour responsable des coûts engagés dans l'activité de vente, mais non pour les coûts induits par la production elle-même.

Ces quatre types fondamentaux de centres à responsabilité comptable sont des modèles théoriques. K. A. Merchant indique qu'il est possible que, bien qu'un dirigeant soit tenu pour responsable d'un centre de responsabilité particulier, il ne soit pas être soumis à l'ensemble des formes de responsabilité qu'emporte tel ou tel type de centre ${ }^{26}$. Il identifie, au sein de la catégorie des centres de profit, quatre différents sous-types, depuis la marge brute jusqu'au « centre de profit complet » avec un niveau croissant de responsabilisation de la direction. Pour évaluer la position spécifique d'une entité au sein de la classification des centres à responsabilité comptable, K. A. Merchant utilise différents aspects des comptes de résultat et des bilans. Le tableau $\mathrm{n}^{\mathrm{o}} 1$ montre les indicateurs utilisés par K. A. Merchant 27.

23. Anthony (R.N.) et Young (D.W.), Management control in non profit organizations, Irwin/ McGrawHill, Boston, 1999, p. 449.

24. Drury (C.), Management and cost accounting, op. cit., p. 655.

25. Merchant (K.A.), Modern management control systems, op. cit., p. 305.

26. Ibidem, p. 306.

27. Avant même d'adapter au secteur public la description que Merchant fait des centres de responsabilité, il convient de faire un commentaire. Dans un centre de profit, le management doit gagner de l'argent en vendant des biens. Dans un environnement traditionnel où paiement et transfert de biens s'opèrent simultanément, c'est une situation logique. Toutefois aujourd'hui, de nombreux biens sont consignés avant que 
Tableau $n^{\circ} 1$ : Éléments pour caractériser les centres à responsabilité comptable (adapté de Merchant ${ }^{28}$ )

\begin{tabular}{|c|c|c|c|c|c|c|c|c|}
\hline & $\begin{array}{l}\text { Centre } \\
\text { de } \\
\text { revenu }\end{array}$ & $\begin{array}{l}\text { Centre } \\
\text { de } \\
\text { dépense } \\
\text { discré- } \\
\text { tionnaire }\end{array}$ & $\begin{array}{c}\text { Centre } \\
\text { de } \\
\text { dépense } \\
\text { - coût } \\
\text { standard }\end{array}$ & $\begin{array}{l}\text { Centre } \\
\text { de } \\
\text { dépense } \\
\text { - coût } \\
\text { complet }\end{array}$ & $\begin{array}{l}\text { Centre } \\
\text { de profit } \\
\text { - marge } \\
\text { brute }\end{array}$ & $\begin{array}{l}\text { Centre } \\
\text { de profit } \\
\text { complet }\end{array}$ & $\begin{array}{l}\text { Centre } \\
\text { d'inves- } \\
\text { tissement } \\
\text { partiel }\end{array}$ & $\begin{array}{l}\text { Centre } \\
\text { d'inves- } \\
\text { tissement } \\
\text { complet }\end{array}$ \\
\hline \multicolumn{9}{|l|}{ État des revenus } \\
\hline Revenu & $\mathrm{x}$ & & & & $\mathrm{x}$ & $\mathrm{x}$ & $\mathrm{x}$ & $\mathrm{x}$ \\
\hline $\begin{array}{l}\text { Coût des biens } \\
\text { vendus }\end{array}$ & & & $\mathrm{x}$ & $\mathrm{x}$ & $\mathrm{x}$ & $\mathrm{x}$ & $\mathrm{x}$ & $\mathrm{x}$ \\
\hline Marge brute & & & & & $\mathrm{x}$ & $\mathrm{x}$ & $\mathrm{x}$ & $\mathrm{x}$ \\
\hline $\begin{array}{l}\text { Publication - } \\
\text { Communication }\end{array}$ & & $\mathrm{x}$ & & $\mathrm{x}$ & & $\mathrm{x}$ & $\mathrm{x}$ & $\mathrm{x}$ \\
\hline $\mathrm{R} \& \mathrm{D}$ & & $\mathrm{x}$ & & $\mathrm{x}$ & & $\mathrm{x}$ & $\mathrm{x}$ & $\mathrm{x}$ \\
\hline Profit & & & & & & $\mathrm{x}$ & $\mathrm{x}$ & $\mathrm{x}$ \\
\hline Profit après impôt & & & & & & $\mathrm{x}$ & $\mathrm{x}$ & $\mathrm{x}$ \\
\hline \multicolumn{9}{|l|}{ BILAN } \\
\hline Effets à recevoir & & & & & & & $\mathrm{x}$ & $\mathrm{x}$ \\
\hline Inventaire & & & & & & & $\mathrm{x}$ & $\mathrm{x}$ \\
\hline $\begin{array}{l}\text { Actifs } \\
\text { immobilisés }\end{array}$ & & & & & & & $\mathrm{x}$ & $\mathrm{x}$ \\
\hline Effets à payer & & & & & & & $\mathrm{x}$ & $\mathrm{x}$ \\
\hline Dette & & & & & & & $\mathrm{x}$ & $\mathrm{x}$ \\
\hline $\begin{array}{l}\text { Résultat net rap- } \\
\text { porté au total } \\
\text { bilan (ROA) }\end{array}$ & & & & & & & & $\mathrm{x}$ \\
\hline
\end{tabular}

\section{LA COMPTABILITÉ PAR CENTRES À RESPONSABILITÉ COMPTABLE DANS LE SECTEUR PUBLIC}

Si nous cherchons maintenant à classifier les agences de la même manière, il convient d'opérer quelques adaptations.

Premièrement, les profits après impôt peuvent être exclus puisque, en général, seules les entités commerciales (for-profit) sont sujettes à l'impôt sur les entreprises.

Deuxièmement, il conviendrait de parler de «coûts de la prestation » au lieu de « coûts des biens vendus ». Dans de nombreux cas, la prestation d'une administration est

le paiement n'ait été effectué ce qui produit une «comptabilité clients ». De plus, la production et les ventes devraient d'une manière ou d'une autre être liées ou, autrement dit, les stocks de produits finis ne devraient pas excéder le niveau qui a été budgété. Si les dirigeants d'un centre de profit sont seulement tenus pour responsables des éléments qui sont dans la présentation des comptes, cela risque de constituer une incitation à générer des revenus sans évaluer de manière critique si la marge qui est censée en résulter sera effectivement réalisée. Ce problème peut être facilement prévenu en donnant au responsable d'un centre de profit la responsabilité du fonds de roulement de l'entité dont il a la charge.

28. Ibid, p. 303-307. 
produite sur un quasi-marché au sein duquel l'accord sur la qualité de service (service level agreement), la décision budgétaire et les coûts constituent les principaux instruments de contrôle. Les ventes peuvent être considérées comme le résultat d'une demande préalable et, le plus souvent, fixée à un prix prédéfini par le commanditaire. Quand aucune relation ne peut être identifiée entre les coûts et les revenus, la notion de "coûts de l'activité » constitue un qualificatif plus approprié.

Troisièmement, la publicité et les campagnes de promotion ont un autre sens dans un environnement public que dans le secteur privé. Le marketing mobilisé pour acquérir de nouveaux clients n'existe en fait que lorsque les administrations peuvent entrer en concurrence soit avec les entités du marché, soit entre elles. Mais la plupart des administrations ont des missions de monopole bien définies d'où la concurrence est exclue. Dans ces cas, la communication en direction des utilisateurs finals (clients / consommateurs) a pour but de donner des informations sur les programmes et les fonctions remplies par l'organisme public. La communication a donc pour but d'informer les destinataires du programme gouvernemental sur la manière de l'utiliser. Nous souhaiterions qualifier cet aspect de la communication de «programme d'information » plutôt que de «publicité et communication » qui est fortement connoté en marketing. Un raisonnement du même type s'applique aux unités de recherche et développement. La recherche et le développement renvoie à la conception de nouveaux produits dans le but de les vendre sur un marché. Quand une administration est autorisée à développer et à mettre en œuvre de nouveaux produits, on peut considérer que cela s'apparente à ce qu'est la recherche et le développement dans un environnement de marché. Si, cependant, l'organisme public est seulement autorisé à commenter les propositions faites par les autres parties pour modifier ou développer de nouveaux produits, alors cela doit être simplement considéré comme une tâche de consultation incluse dans les coûts de l'activité et dans le coût de la prestation produite. Nous utiliserons l'expression «développement de produit autonome» pour indiquer ces activités qui pourraient être labellisées comme recherche et le développement dans un environnement commercial.

Enfin, quatrièmement, la notion de « revenus » peut être ambiguë dans le secteur public puisqu'elle peut également signifier revenu généré par l'impôt. Là où la budgétisation des dépenses emporte qu'un plafond soit fixé pour les dépenses réalisées au cours d'un cycle budgétaire, la part des revenus tirés de l'impôt est le plus souvent considérée comme l'estimation la plus probable de revenu attendu et ne constitue pas un objectif à atteindre. De plus, se focaliser sur la comptabilité par centre de responsabilité implique de se pencher sur les activités de l'organisme public. Quand elles se réalisent sous la forme de transactions financières, c'est la qualité et le volume des transactions qui doivent être évalués. C'est seulement dans les cas où la direction de l'organisme se voit aussi assigner la tâche spécifique de contrôler les coûts de programme que la comptabilité par centre de responsabilité peut y être aussi étendue. Un centre d'impôt peut être considéré, pour l'administration dans son ensemble, comme un centre de revenu, le financement réel de l'organisation et l'accord de qualité de service (service level agreement) déterminant la manière dont les activités de cette entité doivent être classifiées. Les organisations du secteur public qui fournissent une prestation en échange d'une prise en charge ou d'un prix dont s'acquitte un usager ${ }^{29}$ sont tenues pour responsables du revenu généré en retour de la livraison de leur prestation ${ }^{30}$. Du point de vue de la comptabilité des centres à

29. Wagner (R.E.) ed., Charging for government, Routledge, Londres, 1991.

30. Minderman (G.D.), Tweede Kamer en Rijksfinanciën. Een studie naar parlementaire sturing van rijksfinanciën in Nederland, Boom Juridische uitgevers, La Haye, 2000, p. 102. 
responsabilité comptable, cela correspond au revenu généré par un service de ventes dans un environnement commercial. Nous utiliserons donc le terme de «financement» pour indiquer les revenus qui constituent directement les ressources dans l'organisation. Le mot « impôts » sera utilisé pour parler des prélèvements obligatoires acquittés sans qu'il y ait livraison immédiate d'un service en retour. Cela peut inclure les impôts mais aussi les charges sociales et les amendes. À partir de ces remarques, on peut construire, comme dans le tableau $\mathrm{n}^{\mathrm{o}} 2$, un schéma ajusté permettant d'évaluer, dans un environnement de secteur public, le type de centre de responsabilité.

Tableau $n^{\circ} 2$ : Classification ajustée des centres à responsabilité comptable

\begin{tabular}{|c|c|c|c|c|c|c|c|c|c|}
\hline & $\begin{array}{l}\text { Centre } \\
\text { de } \\
\text { revenu } \\
\text { complet }\end{array}$ & $\begin{array}{l}\text { Centre } \\
\text { de } \\
\text { revenu } \\
\text { partiel }\end{array}$ & $\begin{array}{l}\text { Centre } \\
\text { de } \\
\text { dépense } \\
\text { discré- } \\
\text { tion- } \\
\text { naire }\end{array}$ & $\begin{array}{c}\text { Centre } \\
\text { de } \\
\text { dépense } \\
\text { - coût } \\
\text { stan- } \\
\text { dard }\end{array}$ & $\begin{array}{l}\text { Centre } \\
\text { de } \\
\text { dépense } \\
\text { - coût } \\
\text { complet }\end{array}$ & $\begin{array}{c}\text { Centre } \\
\text { de } \\
\text { profit - } \\
\text { marge } \\
\text { brute }\end{array}$ & $\begin{array}{l}\text { Centre } \\
\text { de } \\
\text { profit } \\
\text { complet }\end{array}$ & $\begin{array}{c}\text { Centre } \\
\text { d'inves- } \\
\text { tisse- } \\
\text { ment } \\
\text { partiel }\end{array}$ & $\begin{array}{c}\text { Centre } \\
\text { d'inves- } \\
\text { tisse- } \\
\text { ment } \\
\text { complet }\end{array}$ \\
\hline \multicolumn{10}{|l|}{ État des revenus } \\
\hline Financement & $\mathrm{x}$ & & & & & $\mathrm{x}$ & $\mathrm{x}$ & $\mathrm{x}$ & $\mathrm{x}$ \\
\hline Impôts & & $\mathrm{x}$ & & & & & & & \\
\hline $\begin{array}{l}\text { Coût de la presta- } \\
\text { tion }\end{array}$ & & $\mathrm{x}$ & & $\mathrm{x}$ & $\mathrm{x}$ & $\mathrm{x}$ & $\mathrm{x}$ & $\mathrm{x}$ & $\mathrm{x}$ \\
\hline Coûts de l'activité & & & $\mathrm{x}$ & & & & & & \\
\hline Marge brute & & & & & & $\mathrm{x}$ & $\mathrm{x}$ & $\mathrm{x}$ & $\mathrm{x}$ \\
\hline $\begin{array}{l}\text { Programme d'in- } \\
\text { formation }\end{array}$ & & & $\mathrm{x}$ & & $\mathrm{x}$ & & $\mathrm{x}$ & $\mathrm{x}$ & $\mathrm{x}$ \\
\hline $\begin{array}{l}\text { Dév. de produit } \\
\text { autonome }\end{array}$ & & & & & & & $\mathrm{x}$ & $\mathrm{x}$ & $\mathrm{x}$ \\
\hline Profit & & & & & & & $\mathrm{x}$ & $\mathrm{x}$ & $\mathrm{x}$ \\
\hline \multicolumn{10}{|l|}{ Bilan } \\
\hline Effets à recevoir & & & & & & $\mathrm{x}$ & $\mathrm{x}$ & $\mathrm{x}$ & $\mathrm{x}$ \\
\hline Inventaire & & & & & & $\mathrm{x}$ & $\mathrm{x}$ & $\mathrm{x}$ & $\mathrm{x}$ \\
\hline $\begin{array}{l}\text { Actifs } \\
\text { immobilisés }\end{array}$ & & & & & & & & $\mathrm{x}$ & $\mathrm{x}$ \\
\hline Effets à payers & & & & & & $\mathrm{x}$ & $\mathrm{x}$ & $\mathrm{x}$ & $\mathrm{x}$ \\
\hline Dette & & & & & & & & $\mathrm{x}$ & $\mathrm{x}$ \\
\hline $\begin{array}{l}\text { Résultat net rap- } \\
\text { porté au total bi- } \\
\text { lan (ROA) }\end{array}$ & & & & & & & & & $\mathrm{x}$ \\
\hline
\end{tabular}

K. A. Merchant montre qu'il n'y a que pour les centres d'investissement qu'un bilan complet est requis pour évaluer les responsabilités. Cela peut conduire à utiliser la comptabilité d'engagement dans une autre perspective. La question est plutôt de savoir si nous sommes en mesure d'identifier des organisations au niveau de l'administration centrale susceptibles de constituer des centres d'investissement. On le sait, la théorie du 
droit de propriété décompose celui-ci en trois éléments : usus, fructus, et abusus ${ }^{31}$. C'est seulement dans le cas de l'abusus que l'entité est entièrement libre d'utiliser, d'acheter et de vendre un bien sans l'assentiment préalable d'une tierce partie. Dans le secteur public, le droit d'abusus n'existe pas. Un contrôle politique et budgétaire y sera en effet exercé. A. Likiermann ${ }^{32}$ défend ainsi le contrôle politique sur ces investissements par l'argument de leur nécessaire protection. À l'inverse du modèle britannique de contrôle politique des investissements, l'Australie utilise un instrument de contrôle administratif au travers de l'approbation par le ministère des finances ${ }^{33}$. Aux Pays-Bas, le contrôle des investissements des agences gouvernementales est effectué dans le cadre des procédures budgétaires standards, en autorisant un budget d'investissement séparé au moment où les budgets des administrations et les budgets de fonctionnement des agences gouvernementales sont adoptés. Les commentaires de A. Likierman et M. Robinson impliquent que, même dans les pays où le budget et la comptabilité d'engagement sont les plus développés, le contrôle des investissements demeure un enjeu. Il est donc peu probable qu'une administration puisse être classée comme un «centre d'investissement complet».

Dans un environnement commercial, les actifs sont une part essentielle du processus de production. En revanche, dans le secteur public, les actifs sont particulièrement difficiles à évaluer (monuments historiques, etc.) et ne sont pas censés être productifs. Les agences gouvernementales qui ont la responsabilité de les gérer ne peuvent pas être classées comme des centres d'investissement. Dans la plupart des cas, il faudra conclure qu'elles constituent en fait des centres de dépense qui récupèrent une partie de leurs coûts par des revenus. Pour ce qui tient au fonds de roulement, la question est la suivante : quel type de responsabilité reste-t-il pour le dirigeant d'une agence gouvernementale ? S'il est tenu pour responsable des créances et des dettes, cela peut signifier qu'il dirige un centre de profit. L'exemple australien montre que, même dans ce pays, les budgets sont encore un mélange de financement de base et de financement par les prestations qui, dans les termes d'une comptabilité par centre de responsabilité, pourrait être traduit par une combinaison entre un centre de dépense discrétionnaire et un centre de coût standard ou centre de profit ${ }^{34}$. C'est seulement pour certaines formes spécifiques de centres de coûts ou de profit que la comptabilité d'engagement peut permettre de produire des informations budgétaires et comptables pertinentes.

Notre hypothèse est que, compte tenu de l'hétérogénéité des agences gouvernementales, les différences qui touchent à la part de responsabilité dans les résultats devraient se refléter dans le type de comptabilité et dans les modes de contrôle.

Nous allons évaluer les responsabilités des agences gouvernementales au travers du cadre adapté de comptabilité par centres à responsabilité comptable présenté au tableau $n^{\circ} 2$. Une analyse plus sophistiquée fondée sur des « tableaux de bord prospectifs » n'a pas été réalisée. Seules deux des agences gouvernementales qui ont été étudiées utilisaient explicitement ce modèle dans leur rapport annuel. Il y a là au moins une indication du fait que les actionnaires des agences gouvernementales ne considèrent pas que des instruments

31. Künneke (R.W.), «Verzelfstandiging bezien vanuit de property rights theorie » in Mol (N.P.) and Verbon H.A.A., ed., Neo-institutionele economie en openbaar bestuur. Perspectieven op de verzelfstandiging van overheidsdiensten, $2^{\mathrm{e}}$ herziene druk, Vuga, La Haye, 1997, p. 17-36

32. Likierman (A.), «Planning and controlling UK public expenditure on a resource bases », Public Money and Management, Vol. 23, n ${ }^{\circ} 1,2003$, p. 46.

33. Robinson (M.), «Financial control in Australian Government Budgeting », Public Budgeting and Finance, Vol. 22, $\mathrm{n}^{\circ} 1,2002$, p. 85.

34. Robinson (M.), «Output-purchase funding and budgeting systems in the public sector», Public Budgeting and Finance, Vol. 22. no 4, 2002, p. 24. 
de contrôle plus sophistiqués soient nécessaires. Un effort pour classifier les indicateurs de performance utilisés par les rapports annuels des agences montre que la perspective de «l'apprentissage » est fortement sous-représentée.

\section{CLASSIFICATION DES AGENCES PAR TYPE DE CENTRE À RESPONSABILITÉ COMPTABLE}

Dans un premier temps, nous considérons le financement comme un indicateur du niveau de contrôle budgétaire en rapport avec la classification des tâches présentées par A. Smullen et S. van Thiel ${ }^{35}$. Ensuite, nous classerons les agences gouvernementales sur la base du cadre d'analyse présenté au tableau $\mathrm{n}^{\circ} 2$. Puis, nous analyserons les différences rencontrées. Enfin, nous tenterons de tirer quelques conclusions touchant à la comptabilité et au contrôle dans les différents types de centres à responsabilité comptable qu'impliquent le statut d'agence de l'administration centrale des Pays-Bas. Pour commencer, nous avons établi une liste de toutes les agences de l'administration centrale des Pays-Bas existant en 2004. L'année 2004 est en effet l'année la plus récente pour laquelle les rapports annuels étaient disponibles au début de cette recherche en mars 2006. Nous les avons tout d'abord classées en fonction, premièrement du type d'activité effectuée (direction opérationnelle des données ou des actifs corporels, transferts de revenus, contrôle/inspection, recherche et production d'informations, délivrance de licences et de permis, police et autres) et, deuxièmement de la part respective des financements public et privé dans l'activité de l'agence en question.

Tableau $n^{o} 3$ : Activités et financements des agences gouvernementales

\begin{tabular}{|c|c|c|c|c|c|c|c|}
\hline & \multicolumn{6}{|c|}{ Financement } & \multirow[t]{2}{*}{ Total } \\
\hline Activités & $\begin{array}{l}\text { Adminis- } \\
\text { tration } \\
\text { de tutelle }\end{array}$ & $\begin{array}{c}\text { Entièrement } \\
\text { public }\end{array}$ & $\begin{array}{l}\text { Public à } \\
\text { plus de } \\
75 \%\end{array}$ & $\begin{array}{l}\text { Privé entre } \\
25 \text { et } 50 \%\end{array}$ & $\begin{array}{l}\text { Privé à } \\
\text { plus de } \\
50 \%\end{array}$ & $\begin{array}{c}\text { Entièrement } \\
\text { privé }\end{array}$ & \\
\hline Gestion (G) & 0 & 8 & 2 & 0 & 1 & 0 & 11 \\
\hline $\begin{array}{l}\text { Transfert de } \\
\text { revenu (TR) }\end{array}$ & 0 & 5 & 0 & 0 & 0 & 0 & 5 \\
\hline $\begin{array}{l}\text { Inspection/ } \\
\text { contrôle (I) }\end{array}$ & 1 & 0 & 0 & 2 & 0 & 0 & 3 \\
\hline Recherche (R) & 0 & 2 & 2 & 2 & 0 & 0 & 6 \\
\hline $\begin{array}{l}\text { Brevets - } \\
\text { licences (B-L) }\end{array}$ & 0 & 0 & 3 & 0 & 0 & 1 & 4 \\
\hline Autres (A) & 0 & 0 & 0 & 0 & 1 & 0 & 1 \\
\hline Police (P) & 0 & 0 & 1 & 0 & 0 & 0 & 1 \\
\hline Total & 1 & 15 & 8 & 4 & 2 & 1 & 31 \\
\hline
\end{tabular}

On apprend ainsi du tableau $\mathrm{n}^{\mathrm{O}} 3$ que la moitié des agences est financée par les budgets publics. On remarque que l'activité de contrôle et d'inspection est dans une certaine mesure financée par des contributions privées, au contraire de ce à quoi on aurait pu s'attendre.

35. Smullen (A.) et Thiel (S. van), Agentschappen : eenheid in verscheidenheid, op. cit., p. 36-44. 
Les rapports annuels pour l'année 2004, en général publiés sur internet, ont servi de base pour classer les agences du point de vue des caractéristiques et des exigences de leur comptabilité, et pour les évaluer dans les termes et les catégories identifiées dans le tableau $\mathrm{n}^{\circ}$ 2. Dans certains cas, aucun rapport annuel (spécifique) n'a été publié. Dans tous les cas en revanche, l'information est intégrée dans les rapports fournis à leurs maître d'ouvrages principaux (à savoir les différentes administrations centrales des Pays-Bas). Pour l'analyse sommaire des contenus, nous avons négligé les informations tirées des budgets et des rapports annuels des administrations. Bien qu'on trouve dans ces documents des données sur les agences gouvernementales, ils restent cantonnés le plus souvent à un bilan, une présentation des comptes et de la trésorerie dans le cadre de notes relatives aux finances. Nous avons d'ailleurs appris des fonctionnaires de l'administration des finances que la présentation d'informations dans le cadre des rapports annuels était délibérément limitée à des données financières factuelles. À leurs yeux, cela n'apparaissait pas rétrospectivement comme un bon choix et, dans le futur, les rapports des différentes administrations incluront davantage d'informations relatives à leur performance. Les données financières fournies sont suffisantes pour se faire une idée du financement et du type de centre de responsabilité, mais pas davantage ${ }^{36}$. Le tableau $n^{\circ} 4$ présente nos résultats en faisant référence, à l'intérieur des parenthèses, aux catégories du tableau $\mathrm{n}^{\mathrm{O}} 3$.

Tableau $n^{\circ} 4$ : Classement des agences gouvernementales des Pays-Bas par centres à responsabilité comptable

\begin{tabular}{|c|c|c|c|c|c|c|c|c|c|}
\hline & $\begin{array}{l}\text { Centre } \\
\text { de } \\
\text { revenu } \\
\text { complet }\end{array}$ & $\begin{array}{l}\text { Centre } \\
\text { de } \\
\text { revenu } \\
\text { partiel }\end{array}$ & \begin{tabular}{|l} 
Centre \\
de \\
dépense \\
discré- \\
tionnaire
\end{tabular} & $\begin{array}{c}\text { Centre } \\
\text { de } \\
\text { dépense } \\
\text { - coût } \\
\text { standard }\end{array}$ & $\begin{array}{c}\text { Centre } \\
\text { de } \\
\text { dépense } \\
\text { - coût } \\
\text { complet }\end{array}$ & $\begin{array}{l}\text { Centre } \\
\text { de } \\
\text { profit - } \\
\text { marge } \\
\text { brute }\end{array}$ & $\begin{array}{c}\text { Centre } \\
\text { de } \\
\text { profit } \\
\text { complet }\end{array}$ & $\begin{array}{c}\text { Centre } \\
\text { d'inves- } \\
\text { tissement } \\
\text { partiel }\end{array}$ & $\begin{array}{c}\text { Centre } \\
\text { d'inves- } \\
\text { tissement } \\
\text { complet }\end{array}$ \\
\hline $\begin{array}{l}\text { Neth Forensic } \\
\text { Inst (R) }\end{array}$ & & & $\mathrm{x}$ & & & & & & \\
\hline $\begin{array}{l}\text { Centr archives } \\
\text { selection }(G)\end{array}$ & & & $\mathrm{x}$ & & & & & & \\
\hline $\begin{array}{l}\text { EVD (relations } \\
\text { pub.) }(\mathrm{R})\end{array}$ & & & $\mathrm{x}$ & & & & & & \\
\hline $\begin{array}{l}\text { IWI (travail et } \\
\text { revenu) }(\mathrm{I}-\mathrm{C} \text { ) }\end{array}$ & & & $\mathrm{x}$ & & & & & & \\
\hline $\begin{array}{l}\text { KLPD (services } \\
\text { centraux de } \\
\text { police) (P) }\end{array}$ & & & $\mathrm{x}$ & & $\mathrm{x}$ & & & & \\
\hline $\begin{array}{l}\text { IND } \\
\text { (immigration) } \\
(B-L)\end{array}$ & & & & $\mathrm{x}$ & & & & & \\
\hline $\begin{array}{l}\text { DJI (prisons) } \\
\text { (G) }\end{array}$ & & & & $\mathrm{x}$ & & & & & \\
\hline
\end{tabular}

36. Les six agences qui n'ont pas de rapport annuel sur internet ne sont pas intégrées dans les analyses qui suivent. Dans plusieurs cas, nous avons dû chercher des données additionnelles (sur les sites internet) pour parvenir à une classification adéquate, notamment pour tout ce qui touche aux agences qui effectuent des activités diverses (mixtes du point de vue de notre taxinomie). 


\begin{tabular}{|c|c|c|c|c|c|c|c|c|c|}
\hline & $\begin{array}{l}\text { Centre } \\
\text { de } \\
\text { revenu } \\
\text { complet }\end{array}$ & $\begin{array}{l}\text { Centre } \\
\text { de } \\
\text { revenu } \\
\text { partiel }\end{array}$ & $\begin{array}{c}\text { Centre } \\
\text { de } \\
\text { dépense } \\
\text { discré- } \\
\text { tionnaire }\end{array}$ & $\begin{array}{l}\text { Centre } \\
\text { de } \\
\text { dépense } \\
\text { - coût } \\
\text { standard }\end{array}$ & $\begin{array}{l}\text { Centre } \\
\text { de } \\
\text { dépense } \\
\text { - coût } \\
\text { complet }\end{array}$ & $\begin{array}{c}\text { Centre } \\
\text { de } \\
\text { profit - } \\
\text { marge } \\
\text { brute }\end{array}$ & $\begin{array}{l}\text { Centre } \\
\text { de } \\
\text { profit } \\
\text { complet }\end{array}$ & $\begin{array}{c}\text { Centre } \\
\text { d'inves- } \\
\text { tissement } \\
\text { partiel }\end{array}$ & $\begin{array}{l}\text { Centre } \\
\text { d'inves- } \\
\text { tissement } \\
\text { complet }\end{array}$ \\
\hline $\begin{array}{l}\text { CJIB (amendes) } \\
\text { (TR) }\end{array}$ & & & & $\mathrm{x}$ & & & & & \\
\hline $\begin{array}{l}\text { IVOP (service } \\
\text { de la paye) }(\mathrm{G})\end{array}$ & & & & & $\mathrm{X}$ & & & & \\
\hline $\begin{array}{l}\text { ITO (services } \\
\text { ict) }(\mathrm{G})\end{array}$ & & & & & $\mathrm{X}$ & & & & \\
\hline $\begin{array}{l}\text { RAD (archives } \\
\text { nationales) (G) }\end{array}$ & & & & & $\mathrm{X}$ & & & & \\
\hline $\begin{array}{l}\text { VWA (agence } \\
\text { alimentation) (I } \\
-\mathrm{C} \text { ) }\end{array}$ & & & & & $\mathrm{x}$ & & & & \\
\hline $\begin{array}{l}\text { SENTER } \\
\text { (innovation) } \\
\text { (TR) }\end{array}$ & & & & & $\mathrm{X}$ & & & & \\
\hline $\begin{array}{l}\text { PD (recherche } \\
\text { maladie flore) } \\
\text { (C- I) }\end{array}$ & & & & & $\mathrm{x}$ & & & & \\
\hline $\begin{array}{l}\text { DR (régulation } \\
\text { de l'agriculture) } \\
\text { (TR) }\end{array}$ & & & & & $\mathrm{X}$ & & & & \\
\hline $\begin{array}{l}\text { DLG } \\
\text { (développement } \\
\text { rural) }(\mathrm{G})\end{array}$ & & & & & $\mathrm{X}$ & & & & \\
\hline $\begin{array}{l}\text { RIVM (santé et } \\
\text { environnement) } \\
\text { (R) }\end{array}$ & & & & & $\mathrm{X}$ & & & & \\
\hline $\begin{array}{l}\text { DGWT } \\
\text { (défense } \\
\text { immobilier) (G) }\end{array}$ & & & & & $\mathrm{X}$ & & & & \\
\hline $\begin{array}{l}\text { BPR (système } \\
\text { d'information } \\
\text { citoyen) }(G)\end{array}$ & & & & & $\mathrm{X}$ & $\mathrm{X}$ & & & \\
\hline $\begin{array}{l}\text { TELECOM } \\
\text { authority }(\mathrm{G})\end{array}$ & & & & & $\mathrm{X}$ & $\mathrm{x}$ & & & \\
\hline $\begin{array}{l}\text { DTO (defence } \\
\text { telematics) }(\mathrm{G})\end{array}$ & & & & & & $\mathrm{x}$ & & & \\
\hline $\begin{array}{l}\text { OCTR (brevets) } \\
(\mathrm{B}-\mathrm{L})\end{array}$ & & & & & & $\mathrm{X}$ & & & \\
\hline $\begin{array}{l}\text { NVI (vaccins) } \\
\text { (O) }\end{array}$ & & & & & & $\mathrm{X}$ & & & \\
\hline $\begin{array}{l}\text { CBG } \\
\text { (évaluation } \\
\text { médicament) (B } \\
- \text { L) }\end{array}$ & & & & & & $\mathrm{X}$ & & & \\
\hline $\begin{array}{l}\text { RGD } \\
\text { (immobilier) } \\
\text { (G) }\end{array}$ & & & & & & & & $\mathrm{X}$ & \\
\hline
\end{tabular}


Dans trois cas, nous ne sommes pas parvenus à identifier les caractéristiques générales du centre de responsabilité en question du fait de son hétérogénéité interne. L'agence KLPD effectue ainsi des services de police générale au niveau national. Cela comporte les responsabilités d'un centre de dépense. Mais elle soutient également les activités des forces de police régionalisées qui, elles, relèvent pleinement d'un centre de coût. L'agence $B P R$ administre pour le gouvernement le système d'information de base des citoyens (ce qui est caractéristique d'un centre de coût); dans le même temps, il délivre des passeports (ce qui est caractéristique d'un centre de profit). De même, l'agence Telecom Authority remplit des missions d'inspection (centre de coût) et délivre des licences (centre de profit).

\section{ANALYSE DES CARACTÉRISTIQUES COMPTABLES DES AGENCES}

Nous sommes parvenus à classer 25 des 31 agences gouvernementales par type de centre de responsabilité. Dans le cas où une agence constituait un ensemble composite et hétérogène, nous l'avons classée dans la catégorie la plus basse des centres de responsabilité. Cela signifie par exemple que l'agence $B P R$ est classée comme un « centre de coût complet » plutôt que comme un «centre de profit à marge brute ». L'étape suivante de l'analyse consiste à comparer cette classification des centres de responsabilité avec une classification fondée sur les tâches de l'organisme.

\section{Tableau $n^{\circ} 5$ : Centres à responsabilité comptable par type de tâches}

\begin{tabular}{|c|c|c|c|c|c|c|}
\hline \multirow[b]{2}{*}{ Activité } & \multicolumn{5}{|c|}{ Centre de responsabilité } & \multirow[t]{2}{*}{ Total } \\
\hline & $\begin{array}{c}\text { Centre de } \\
\text { dépense } \\
\text { discré- } \\
\text { tionnaire }\end{array}$ & $\begin{array}{c}\text { Centre de } \\
\text { dépense } \\
\text {-coût } \\
\text { standard }\end{array}$ & $\begin{array}{c}\text { Centre de } \\
\text { dépense - } \\
\text { coût } \\
\text { complet }\end{array}$ & $\begin{array}{l}\text { Centre de } \\
\text { profit - } \\
\text { marge brute }\end{array}$ & $\begin{array}{c}\text { Centre } \\
\text { d'inves- } \\
\text { tissement } \\
\text { partiel }\end{array}$ & \\
\hline Gestion & 1 & 1 & 6 & 2 & 1 & 11 \\
\hline $\begin{array}{l}\text { Transfert } \\
\text { revenu }\end{array}$ & 0 & 1 & 2 & 0 & 0 & 3 \\
\hline $\begin{array}{l}\text { Inspection / } \\
\text { contrôle }\end{array}$ & 1 & 0 & 2 & 0 & 0 & 3 \\
\hline Recherche & 2 & 0 & 1 & 0 & 0 & 3 \\
\hline $\begin{array}{l}\text { Licences / } \\
\text { brevêts }\end{array}$ & 0 & 1 & 0 & 2 & 0 & 3 \\
\hline Autres & 0 & 0 & 0 & 1 & 0 & 1 \\
\hline Police & 0 & 0 & 1 & 0 & 0 & 1 \\
\hline Total & 4 & 3 & 12 & 5 & 1 & 25 \\
\hline
\end{tabular}

Il résulte de cette comparaison que tous les «centres de profit» et tous les «centres d'investissement » peuvent être classés comme des entités pour lesquelles une production mesurable semble être disponible. Nous partons du principe que l'activité de gestion, celle qui consiste à délivrer des licences et des permis, ou encore celle qui tient dans le transfert 
de revenus, donnent des résultats chiffrables. Ainsi, l'agence NVI, classé dans la catégorie « autres », produit des vaccins, ce qui peut être clairement mesuré. Il reste que les dix-neuf « centres de coût » sont largement répartis sur l'éventail des tâches identifiées. On peut cependant établir que là où la production est plus difficile à mesurer (recherche, inspection, et activité de police), les agences gouvernementales sont toutes classées comme des « centres de coût», ce qui implique une forme de contrôle budgétaire. Les différences du point de vue de la classification des centres de coûts ne peuvent toutefois pas être expliquées par la différenciation des tâches.

Nous avons commencé notre analyse au tableau $n^{\circ} 3$ par une étude des financements et des tâches assignées aux agences gouvernementales. Nous pouvons maintenant appliquer cette analyse au tableau $\mathrm{n}^{\circ} 6$ en tenant désormais compte de notre classification des centres de responsabilité. On pourrait s'attendre à ce que le contrôle des coûts ait un lien avec le financement public. Cette hypothèse est confirmée pour l'essentiel.

Tableau $n^{\circ} 6$ : Financement par type de centres à responsabilité comptable

\begin{tabular}{|l|c|c|c|c|c|c|c|}
\hline \multirow{2}{*}{$\begin{array}{l}\text { Type de centre } \\
\text { de } \\
\text { responsabilité }\end{array}$} & $\begin{array}{c}\text { Adminis- } \\
\text { tration de } \\
\text { tutelle }\end{array}$ & $\begin{array}{c}\text { Entiè- } \\
\text { rement } \\
\text { public }\end{array}$ & $\begin{array}{c}\text { Public à } \\
\text { plus de } \\
75 \%\end{array}$ & $\begin{array}{c}\text { Privé de } \\
25 \text { à } \\
50 \%\end{array}$ & $\begin{array}{c}\text { Privé à } \\
\text { plus de } \\
50 \%\end{array}$ & $\begin{array}{c}\text { Entière- } \\
\text { ment } \\
\text { privé }\end{array}$ & Total \\
\hline $\begin{array}{l}\text { Centre de } \\
\text { dépense } \\
\text { discrétionnaire }\end{array}$ & 1 & 2 & 1 & 0 & 0 & 0 & 4 \\
\hline $\begin{array}{l}\text { Centre de } \\
\text { dépense-coût } \\
\text { standard }\end{array}$ & 0 & 2 & 1 & 0 & 0 & 0 & 3 \\
\hline $\begin{array}{l}\text { Centre de } \\
\text { dépense-coût } \\
\text { complet }\end{array}$ & 0 & 7 & 2 & 3 & 0 & 0 & 12 \\
\hline $\begin{array}{l}\text { Centre de profit } \\
- \text { marge brute }\end{array}$ & 0 & 1 & 1 & 0 & 2 & 1 & 5 \\
\hline $\begin{array}{l}\text { Centre } \\
\text { d'investissement } \\
\text { partiel }\end{array}$ & 0 & 0 & 1 & 0 & 0 & 0 & 1 \\
\hline Total & 1 & 12 & 6 & 3 & 2 & 1 & 25 \\
\hline
\end{tabular}

Des vingt-cinq agences classifiées, dix-neuf ont été catégorisées comme des centres de coût et seize d'entre elles sont financées à plus de $75 \%$ par les budgets publics. De ce fait, pour l'immense majorité des agences, on peut s'interroger sur la pertinence de l'application d'une comptabilité d'engagement. La comptabilité budgétaire qui concerne leur financement risque en effet de neutraliser les améliorations en termes d'efficacité qui sont habituellement associées à l'introduction d'un tel système comptable. Les données disponibles indiquent que onze des agences gouvernementales qui relèvent du type des « centres de coût » ont des actifs immobilisés qui correspondent à plus de $30 \%$ du total de leur budget. Comment de telles agences gouvernementales peuvent-elles utiliser les actifs de manière aussi efficace que possible quand leur financement est tiré du secteur public où la comptabilité budgétaire constitue le principal instrument de contrôle? 
Les données tirées des six autres agences gouvernementales (qui ne sont pas des centres de coût) sont plus ambiguës. Seule la moitié d'entre elles présente un financement privé substantiel. Une analyse plus fine révèle que ces trois agences financées par des entités privées ont des utilisateurs finals (end users) qui se situent en dehors de la sphère de contrôle direct du gouvernement central. Deux autres agences opèrent comme des «centres de service partagé » (shared service centre) pour le compte de l'administration centrale. Cela signifie que la plupart des centres d'investissement et de profit exercent leur activité dans un environnement qui est dominé par l'offre et la demande plutôt que dans un contexte d'un contrôle budgétaire immédiat. Cette conclusion nous conduit à une analyse complète de la relation entre les centres de responsabilité et les utilisateurs finals des services. Il apparaît ainsi que dix-sept des vingt-cinq agences gouvernementales ont des utilisateurs finals en dehors de l'administration centrale elle-même. Bien que celle-ci puisse être le commanditaire des tâches effectuées par l'agence, près de la moitié des agences gouvernementales dépendent exclusivement d'une demande extérieure à l'administration pour produire leurs services. Cette situation implique que les budgets des agences peuvent difficilement être contrôlés par l'administration centrale. Cela est reflété par le fait qu'à une exception près, toutes les agences (voir en gras sur le tableau $\mathrm{n}^{\circ} 7$ ) sont des centres de responsabilité dans lesquels des budgets flexibles doivent être disponibles ${ }^{37}$.

Tabeau $n^{\circ}$ 7: Utilisateurs finals des services des agences par type de centres à responsabilité comptable

\begin{tabular}{|c|c|c|c|c|c|c|}
\hline \multirow[b]{2}{*}{$\begin{array}{c}\text { Type de centre } \\
\text { de } \\
\text { responsabilité }\end{array}$} & \multicolumn{5}{|c|}{ Utilisateur final } & \multirow[b]{2}{*}{ Total } \\
\hline & $\begin{array}{l}\text { Unités } \\
\text { adminis- } \\
\text { tratives }\end{array}$ & $\begin{array}{l}\text { Plus d'une } \\
\text { adminis- } \\
\text { tration }\end{array}$ & $\begin{array}{l}\text { Adminis- } \\
\text { tration ou } \\
\text { autres } \\
\text { entités } \\
\text { publiques }\end{array}$ & $\begin{array}{l}\text { Uniquement } \\
\text { d'autres } \\
\text { entités } \\
\text { publiques }\end{array}$ & $\begin{array}{l}\text { Secteur } \\
\text { privé }\end{array}$ & \\
\hline $\begin{array}{l}\text { Centre de } \\
\text { dépense } \\
\text { discrétionnaire }\end{array}$ & 2 & 0 & 1 & 1 & 0 & 4 \\
\hline $\begin{array}{l}\text { Centre de } \\
\text { dépense-coût } \\
\text { standard }\end{array}$ & 0 & 0 & 2 & $\mathbf{0}$ & 1 & 3 \\
\hline $\begin{array}{l}\text { Centre de } \\
\text { dépense-coût } \\
\text { complet }\end{array}$ & 2 & 3 & 2 & 3 & 2 & 12 \\
\hline $\begin{array}{l}\text { Centre de profit } \\
- \text { marge brute }\end{array}$ & 0 & 0 & 1 & 1 & 3 & 5 \\
\hline $\begin{array}{l}\text { Centre } \\
\text { d'investissement } \\
\text { partiel }\end{array}$ & 0 & 1 & 0 & 0 & 0 & 1 \\
\hline Total & 4 & 4 & 6 & 5 & 6 & 25 \\
\hline
\end{tabular}

37. Les budgets flexibles peuvent être adaptés en fonction des effets de « volume externe », Drury (C.), Management and cost accounting, op. cit., p. 659. 


\section{CONCLUSION INTERMÉDIAIRE}

On a vu qu'il semblait y avoir une correspondance entre les types de centres de responsabilité utilisés et la flexibilité requise quand les centres dépendent complètement de clients extérieurs. On observe aussi une tendance à l'accroissement de la complexité des centres à responsabilité comptable quand le financement tend à être davantage lié au secteur privé. Les financements publics correspondent davantage à des centres à responsabilité comptable qui sont du type « centre de coût ». On peut s'attendre à ce que, particulièrement dans le cas des centres de coût, des instruments de contrôle supplémentaires (non financiers) soient utilisés.

La dernière étape de notre analyse consiste à regarder plus en détail les informations comptables qui sont fournies par les agences gouvernementales dans leurs rapports annuels. Pour ce faire, on s'est concentré sur les indicateurs de performance non financiers tels qu'ils sont présentés par les rapports annuels des agences gouvernementales elles-mêmes. Quand le contrôle budgétaire reste encore directement ou indirectement important, rappelons que $75 \%$ des agences gouvernementales sont presque entièrement financées par des fonds publics, les instruments de contrôle non financiers deviennent plus importants. Nous avons établi une distinction, parmi les indicateurs de moyens ou intrants, entre ceux liés au processus de producteur, et ceux liés à la prestation. Nos données sont présentées tableau $n^{\circ} 8$.

Tableau $n^{\circ} 8$ : Les indicateurs de performance non financiers par catégorie de centres à responsabilité comptable

\begin{tabular}{|l|c|c|c|c|c|c|}
\hline & \multirow{2}{*}{$\begin{array}{c}\text { Nombre } \\
\text { d'agences }\end{array}$} & Moyens & $\begin{array}{c}\text { Processus } \\
\text { de } \\
\text { production }\end{array}$ & Prestation & Résultats & \\
\cline { 3 - 7 } & & 2 & 0 & 0 & 0 & 2 \\
\hline $\begin{array}{l}\text { Centre de } \\
\text { dépense } \\
\text { discrétionnaire }\end{array}$ & 4 & 29 & 29 & 18 & 7 & 83 \\
\hline $\begin{array}{l}\text { Centre de } \\
\text { dépense - coût } \\
\text { standard }\end{array}$ & 3 & 23 & 27 & 28 & 2 & 80 \\
\hline $\begin{array}{l}\text { Centre de } \\
\text { dépense - coût } \\
\text { complet }\end{array}$ & 12 & 16 & 16 & 9 & 0 & 42 \\
\hline $\begin{array}{l}\text { Centre de profit } \\
\text { - marge brute }\end{array}$ & 5 & & & & & \\
\hline $\begin{array}{l}\text { Centre } \\
\text { d'investissement } \\
\text { partiel }\end{array}$ & 1 & 0 & 0 & 7 & 0 & 7 \\
\hline Total & 25 & 70 & 72 & 62 & 10 & 214 \\
\hline
\end{tabular}

La majorité des indicateurs renvoient aux moyens (ou intrants) et au processus de production. On remarquera qu'on ne voit pas de différence concernant les mesures de la performance non financière selon qu'il s'agit d'un centre de coût ou non ; dans les deux cas, environ les deux tiers des indicateurs non financiers sont centrés sur les moyens ou sur le 
processus de production. On pourrait s'attendre à ce que les centres à responsabilité comptable qui ne sont pas liés au coût offrent davantage d'indicateurs de prestation. Mais, ici encore, le résultat de notre recherche semble indiquer que les instruments de contrôle budgétaire restent prépondérants, y compris pour les agences qui sont supposées évoluer de manière relativement indépendante des mécanismes traditionnels de contrôle de l'administration. Les carences qui ressortent de ces résultats en terme de comptabilité apparaissent particulièrement quand on s'interroge pour savoir dans quelle mesure la valeur des indicateurs ex post est comparée avec un quelconque objectif qui avait été fixé ex ante et vice versa.

Tableau $n^{\circ} 9$ : Indicateurs de performance ex ante et ex post par catégorie centres à responsabilité comptable

\begin{tabular}{|l|c|c|c|c|c|c|}
\hline & \multirow{2}{*}{$\begin{array}{c}\text { Nombre } \\
\text { d'agences }\end{array}$} & $\begin{array}{c}\text { Pas } \\
\text { d'objectif }\end{array}$ & $\begin{array}{c}\text { Objectifs } \\
\text { chiffrés ex } \\
\text { ante }\end{array}$ & $\begin{array}{c}\text { Résultats } \\
\text { réalisés ex } \\
\text { post }\end{array}$ & Les deux & \\
\hline $\begin{array}{l}\text { Centre de } \\
\text { dépense } \\
\text { discrétionnaire }\end{array}$ & 4 & 0 & 0 & 2 & 0 & 2 \\
\hline $\begin{array}{l}\text { Centre de } \\
\text { dépense - coût } \\
\text { standard }\end{array}$ & 3 & 8 & 4 & 45 & 26 & 83 \\
\hline $\begin{array}{l}\text { Centre de } \\
\text { dépense - coût } \\
\text { complet }\end{array}$ & 12 & 0 & 0 & 54 & 26 & 80 \\
\hline $\begin{array}{l}\text { Centre de profit } \\
- \text { marge brute }\end{array}$ & 5 & 0 & 0 & 24 & 18 & 42 \\
\hline $\begin{array}{l}\text { Centre } \\
\text { d'investissement } \\
\text { partiel }\end{array}$ & 1 & 0 & 0 & 7 & 0 & 7 \\
\hline Total & 25 & 8 & 3 & 132 & 70 & 214 \\
\hline
\end{tabular}

Le rapport entre les valeurs attendues et les valeurs réalisées n'est en fait présenté dans les rapports annuels uniquement pour $30 \%$ des indicateurs de performance non financière. Dans la grande majorité des cas, il n'y a que les chiffres réalisés ex post. Deux remarques peuvent être formulées à cet égard. Premièrement, dans le cadre du contrôle budgétaire, on pourrait s'attendre à ce qu'une comparaison entre le projet et la réalisation soit effectuée. Ce n'est clairement pas le cas. Pour les hypothèses où l'efficacité semble être la motivation principale qui a poussé à l'adoption d'un statut d'agence (en italique dans le tableau $n^{\circ} 9$ ), $36 \%$ des indicateurs sont à la fois ex ante et ex post. On aurait pu s'attendre ici à ce qu'une attention plus grande soit portée aux seules données ex post. Deuxièmement, la procédure budgétaire néerlandaise se donne aujourd'hui pour but de renforcer l'objectif de performance permettant d'établir un rapport entre les objectifs fixés et les résultats obtenus. Or, il apparaît que les objectifs sont explicitement évoqués uniquement dans un nombre limité de cas, ce qui est contraire à la politique des responsables gouvernementaux. 


\section{ÉVALUATION DES RÉSULTATS}

Dans cet article, nous avons tenté d'évaluer le type de « responsabilité pour résultats » qui est effectivement inscrite dans le système de comptabilité des agences gouvernementales des Pays-Bas. Notre hypothèse générale est que, compte tenu de l'hétérogénéité des agences, les différences concernant le type de responsabilité pour résultats devrait se refléter dans le type de comptabilité et le type de contrôle qui leur est appliqué. On a d'abord classé les agences ( $\mathrm{n}=25)$ dont le rapport annuel pour l'année 2004 était disponible dans le cadre du modèle adapté des centres à responsabilité comptable ( $c f$. tableau $\left.\mathrm{n}^{\circ} 2\right)$. Il en est ressorti, pour l'essentiel, une classification homogène marquée par une forte présence des centres de coût : dix-neuf des vingt-cinq agences sont classées dans cette catégorie. La plupart sont à part entière des centres de coût, ce qui signifie qu'elles sont pleinement responsables pour l'ensemble des coûts de l'agence. On pourrait rétorquer que, si le contrôle de coût constitue bien un enjeu primordial, le management est responsable de l'ensemble des activités et des coûts conformément à l'idée selon laquelle les agences doivent opérer indépendamment de l'administration centrale. Il reste que le contrôle des coûts et le contrôle du budget sont étroitement liés dans le secteur public. Cela incite à s'interroger sur le fait de savoir si la comptabilité d'engagement est véritablement intéressante pour les agences gouvernementales. Nous avons utilisé ici les informations concernant le type de financement et le type d'utilisateurs finals pour évaluer la position des différents types de centre de responsabilité. En fonction du premier résultat, ceux qui ne sont pas des centres de coût tendent à être financés par des fonds privés et ont des utilisateurs finals en dehors de l'administration centrale. Dans ces cas, l'offre et la demande forment un environnement de marché pour lequel l'allocation des dépenses peut être utilement fondée sur une comptabilité d'engagement. Par ailleurs, la majorité des agences relevant de la catégorie «centres de coût » dispose d'une somme importante d'actifs fixes tandis que leur financement est essentiellement public. Il y a ici un conflit potentiel entre l'usage de la comptabilité par engagement et les mécanismes de contrôle de ces entités. Une enquête plus fouillée fondée sur les indicateurs de performance non financiers présentés dans les rapports annuels des agences révèle que deux tiers de ces indicateurs sont centrés sur les prestations ou sur le processus de production, et ce, quel que soit le type de centre de responsabilité. Il y a là une indication du fait que le contrôle de type budgétaire semble dominer. Cette situation est d'autant plus intéressante qu'elle entre en contradiction avec une des règles qui gouvernent la comptabilité publique aux Pays-Bas : cette dernière doit indiquer la relation entre les objectifs et les résultats. L'attention presque exclusive portée à une présentation ex post indique que les objectifs jouent ici en fait un rôle mineur.

On pourrait en conclure que notre étude fournit des résultats ambigus. D'une part, il semble y avoir une correspondance entre le type de centre de responsabilité et un rapport entre l'offre et la demande proche de celui d'un marché. D'autre part, le fait que les indicateurs de performance soient fortement centrés sur l'intrant et le processus de production plutôt que sur les prestations rendues et le résultat semble indiquer un contrôle budgétaire de type traditionnel. À travers une étude de cas approfondie de certaines des agences gouvernementales, on pourrait faire apparaître comment le contrôle est effectivement réalisé de manière à trouver des explications pour ces résultats ambigus. 